\title{
Verfügbarkeit und Leistung - Synthese zweier Kennwerte für die anforderungsgerechte Planung intralogistischer Systeme
}

\author{
Univ.-Prof. Dr. Michael ten Hompel, Dr.-Ing. Volker Sadowsky, \\ Dipl.-Logist. Eike-Niklas Jung, Dipl.-Inform. Christian Mosblech \\ Technische Universität Dortmund \\ Lehrstuhl für Förder- und Lagerwesen
}

\begin{abstract}
Hohe Leistungsfähigkeit bei niedrigen Kosten sowie hoher Servicegrad und Flexibilität bilden die maßgeblichen Zielkriterien für intralogistische Systeme. Die daraus resultierende Anforderung der Systembetreiber nach einer adäquaten Leistungserbringung ist im Wesentlichen als mengen- und zeitgerechte Bewältigung der in das System eingegebenen Last zu verstehen. Bei der Erfüllung dieser dynamischen Anforderungen sind insbesondere drei Faktoren zu berücksichtigen, die einen Einfluss auf die Leistungserbringung besitzen. Hierbei handelt es sich um die technische Verfügbarkeit, die maximale Leistungsfähigkeit (Grenzleistung) sowie die Steuerungsstrategie auf operativer bzw. dispositiver Ebene. Die Untersuchung des Zusammenwirkens dieser Faktoren und die Zusammenführung dieser zu einem integrativen Berechnungsmodell sind Gegenstand aktueller Forschungsarbeiten am Lehrstuhl für Förder- und Lagerwesen. Ziel hierbei ist es, bereits im Rahmen der Systemplanung die zu erwartende, tatsächlich nutzbare Systemleistung zu ermitteln und bei der Systemgestaltung zu berücksichtigen, ohne hierfür aufwändige Simulationsstudien durchführen zu müssen. Der folgende Beitrag fasst die im Rahmen des Sonderforschungsbereich 696 der Deutschen Forschungsgemeinschaft erarbeiteten Ergebnisse aus dem Bereich der technischen Verfügbarkeit zusammen und gibt einen Ausblick auf weitere Arbeiten, die zur Entwicklung des integrierten Berechnungsmodells der Leistungsverfügbarkeit notwendig sind.
\end{abstract}

\section{$1 \quad$ Einleitung}

Eine der wichtigsten Aufgaben bei der Planung intralogistischer Systeme ist die anforderungsgerechte Gestaltung des Systems unter Berücksichtigung erforderliche Leistung. Wesentliche Einflussfaktoren sind in diesem Zusammenhang die Festlegung der Systemstruktur sowie die Auswahl geeigneter Materialflusskomponenten. Aufgrund der Vielfalt der Lösungsmöglichkeiten entstehen hierdurch meist mehrere Varianten, die mit Hilfe entsprechender Verfahren in Bezug auf ihre Leistung bewertet werden müssen. Der Aufwand hierfür sollte in einem angemessenen Verhältnis zum Nutzen stehen. Insbesondere in frühen Phasen der Planung, in denen eine Vielzahl von Varianten zu bewerten ist, sind Methoden gut geeignet, die einen vergleichsweise geringen Modellierungsaufwand mit einer zu diesem Zeitpunkt adäquaten Ergebnisgüte kombinieren.

Um zu einer Aussage über die tatsächlich zu erwartende Leistung zu gelangen, ist neben der technisch bedingten Leistungsfähigkeit der einzelnen Komponenten auch 
ihre (technische) Verfügbarkeit zu berücksichtigen. Letztere gibt Aufschluss über das Verhältnis der tatsächlich nutzbaren zur theoretischen Einsatzzeit des Systems bzw. der verbauten Komponenten. Zur Ermittlung der Verfügbarkeit existiert eine Reihe von Methoden, deren Eigenschaften und einschränkende Randbedingungen in [tHo07] analysiert wurden. Das Ziel dieser Methoden ist es, von der Verfügbarkeit mehrerer Einzelkomponenten zu der eines (Teil-)Systems zu gelangen. Dies geschieht im Wesentlichen durch vorgefertigte Strukturelemente für beispielsweise seriell oder parallel angeordnete Komponenten. Diese Elemente liefern entsprechende Berechnungsvorschriften und ermöglichen somit die Kennwertermittlung für das Teilsystem. Durch erneute Kombination lässt sich somit iterativ die Systemverfügbarkeit ermitteln. Diese Vorgehensweise ist für Systeme mit vergleichsweise einfacher Systemstruktur durchaus praktikabel, stößt jedoch mit zunehmender Systemkomplexität an ihre Grenzen. Je komplexer die Struktur eines Systems wird, umso höher ist die Wahrscheinlichkeit, dass Anordnungen auftreten, die sich mit Hilfe der vorgefertigten Strukturelemente nicht modellieren lassen.

Aus diesen Gründen wurde eine Methode zur realitätsnahen Abbildung der technischen Verfügbarkeit intralogistischer Systeme entwickelt, die im Folgenden vorgestellt und beispielhaft zur Anwendung gebracht wird. Die Methode ermöglicht es, in späten Phasen der Systemplanung und während des Systembetriebs die Verfügbarkeit und mit ihr verbundene Kennwerte zu ermitteln.

Abschließend wird Ausblick auf weitere Arbeiten gegeben, die zur Entwicklung des integrierten Berechnungsmodells der Leistungsverfügbarkeit notwendig sind. Diese fokussieren den Bereich der Leistungsermittlung von Intralogistiksystemen, da die Anwendung der hierfür existierenden Verfahren vielfach auf einzelne Teilsysteme oder Systemelemente beschränkt ist und Wechselwirkungen zwischen einzelnen Systemelementen nicht oder nur unzureichend berücksichtigt werden.

\section{Grundlagen}

Die folgenden Abschnitte geben einen kurzen Überblick über verwandte Arbeiten im Bereich der Verfügbarkeits- und Zuverlässigkeitsanalyse. Desweiteren werden die zum Verständnis der vorgestellten Methode notwendigen theoretischen Grundlagen kurz zusammengefasst. Eine ausführlichere Darstellung findet sich in [tHo09].

Die vorgestellte Methode nutzt zwei Verfahren, die bereits im Kontext von Zuverlässigkeits- und Verfügbarkeitsuntersuchungen eingesetzt wurden. Dies sind zum einen gerichtete Graphen und zum anderen Petri-Netze. Die Graphentheorie dient dabei zur Modellierung der Systemstruktur sowie zur Ermittlung des Systemzustands. Petri-Netze mit einfacher Struktur werden beispielhaft zur Modellierung des Verhaltens der Komponenten eingesetzt.

Der Begriff der technischen Verfügbarkeit (engl. availability) bezeichnet einen Kennwert der Zuverlässigkeitstheorie, der das Verhältnis zwischen der tatsächlichen Laufzeit (Einschaltzeit $T_{E}-$ Ausfallzeit $T_{A}$ ) eines Systems und der Einschaltzeit $\left(T_{E}\right)$ 
angibt. Dieser Wert stellt die Relation zwischen der praktisch nutzbaren zur theoretisch möglichen Leistungsfähigkeit eines Systems dar und ist folglich die einzig geeignete Kennzahl zur Quantifizierung der Einsatzbereitschaft einer technischen Anlage (vgl. [Arn90]). Die Verfügbarkeit basiert auf dem alternierenden Zustandsverhalten instandsetzbarer Komponenten und Systeme, die durch den Wechsel zwischen Intakt- und Defektzustand charakterisiert werden. Ein Zustandswechsel findet dabei durch die Ereignisse des Ausfalls und der Instandsetzung statt. Dieses Verhalten eines instandsetzbaren Systems ist in Abbildung 1 in Abhängigkeit der Zeit dargestellt.

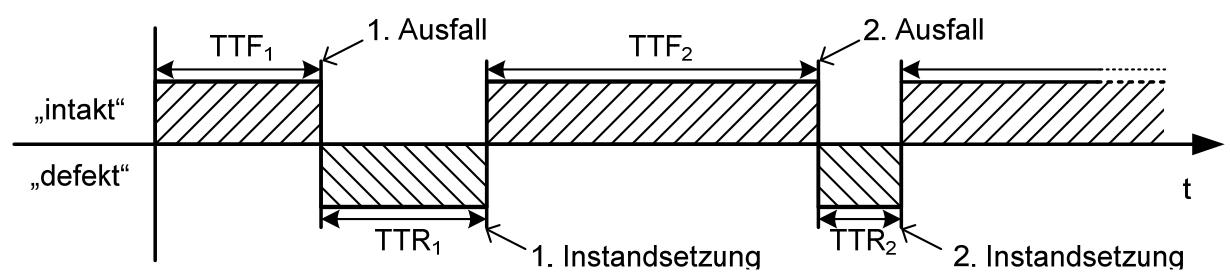

Abbildung 1: Alternierendes Zustandsverhalten instandsetzbarer Systeme (vgl. [Bov00])

Auf dieser Grundlage lässt sich die Verfügbarkeit aus zwei Kennwerten bestimmen. Dies sind zum einen der Mittelwert der ausfallfreien Zeit TTF, der als MTTF (engl. mean time to failure) bezeichnet wird, und zum anderen der Mittelwert der Instandsetzungsdauer TTR, kurz MTTR (engl. mean time to repair). Hieraus lässt sich die Verfügbarkeit als Verhältnis der MTTF zur Summe aus dieser und der MTTR [VDI04] berechnen.

Die Eignung der Graphentheorie zur Ermittlung von Zuverlässigkeitskennwerten wird beispielsweise in [Fau08] gezeigt, wo sie erfolgreich zur Nachbildung der Systemstruktur genutzt wird. Die grundsätzliche Eignung von Petri-Netzen zur Ermittlung von Zuverlässigkeitskennwerten wird beispielsweise in [Poz06] und in [Neb09] gezeigt. Das zeitabhängige Verhalten der Komponenten sowie deren Abhängigkeiten untereinander werden dabei in dieser Form modelliert.

Zur Beschreibung der Systemstruktur kommen gerichtete Graphen zum Einsatz. Jeder Graph $G$ ist dabei bestimmt durch ein 2-Tupel $G=(V, E)$, wobei $V$ die Knotenmenge und $E \subseteq V \times V$ die Kantenmenge darstellt. Als Knoten werden in dem Modell alle technischen Einheiten verstanden, beginnend bei ganzen Systemen, über Subsystemen bis hin zu einzelnen Systemkomponenten, wie z.B. Förderern oder Regalbediengeräten. Die Kanten des Graphen bilden den strukturellen und technischen Zusammenhang der beteiligten Knoten ab und somit auch den möglichen Materialfluss und dessen Richtung.

Petri-Netze sind eine mathematische Modellierungsmethode, die verwendet werden kann, um Systeme und Transformationsprozesse in Systemen formal zu beschreiben und auszuwerten. Sie gehören zur Klasse der zustandsraumorientierten Modelle (vgl. [Poz06]). Informal kann man ein Petri-Netz als endlichen, gerichteten, zweigefärbten Graphen definieren ([Pri08], S.20ff.). Zweigefärbt bedeutet hierbei, dass ein Petri- 
Netz über zwei Knotentypen „Stellen“ (engl. places) und „Transitionen“ (engl. transitions) verfügt, wobei keine Kante zwei Knoten gleichen Typs miteinander verbindet. In der graphischen Darstellung werden Stellen als Kreise und Transitionen als Rechtecke dargestellt. Transitionen repräsentieren eine Aktion (oder auch ein Ereignis) innerhalb des Systems. Stellen dagegen sind mit Speicherzellen vergleichbar und können eine Anzahl von sogenannten Marken (engl. token) aufnehmen.

In diesem Beitrag werden stochastisch zeitbehaftete Petri-Netze zur Abbildung des Verhaltens der Systemkomponenten genutzt. Diese Art von Petri-Netzen eignet sich gut für Zuverlässigkeitsanalysen, da neben Wahrscheinlichkeiten und Wahrscheinlichkeitsverteilungen auch Übergangsraten (hier: Ausfall- bzw. Instandsetzungsrate, vgl. [Bov00]) als Wahrscheinlichkeitsmaßgrößen verwendet werden können. Details zu den weiteren Netztypen sind beispielsweise [Pri08] zu entnehmen.

Zur Modellierung der Komponenten in einem Petri-Netz sind zunächst alle Zustände zu definieren, die diese im Zusammenhang mit ihrem Ausfall- bzw. Instandsetzungsverhalten annehmen kann. Nach [Poz06] lassen sich hierbei operative und inhärente Zustände differenzieren. Die operativen Zustände kennzeichnen den jeweiligen Betriebsstatus der Komponente (z. B. „in Betrieb“ oder „ausgefallen und in Instandsetzung") und lassen sich eindeutig einem inhärenten Zustand zuordnen. Die inhärenten Zustände lassen sich wiederum in "intakt" und "defekt" unterscheiden, wodurch gekennzeichnet wird, ob die Komponente zum betrachteten Zeitpunkt in der Lage ist, die geforderte Funktion zu erfüllen oder nicht. Vereinfachend werden im Folgenden lediglich die inhärenten Zustände betrachtet. Der Übergang zwischen den Zuständen wird durch die Ereignisse „Ausfall“ und „Instandsetzung" beschrieben. Das sich hieraus ergebende Petri-Netz für eine Komponente ist in Abbildung 2 dargestellt.

Das Eintreten dieser Ereignisse wird durch die zeitliche Bewertung von Transitionen erzielt. Bei der Verwendung von stochastischen Netzen lässt sich diese Bewertung durch statistische Verteilungsfunktionen realisieren. Im einfachsten Fall wird für jede Komponente ein eigenes Netz angelegt, das das Verhalten der jeweiligen Komponente nachbildet. Durch schrittweise, zeitdiskrete Berechnung wird anschließend das zeitliche Verhalten ermittelt, wobei sich die Zustandsänderungen aufgrund der definierten Transitionen ergeben. 


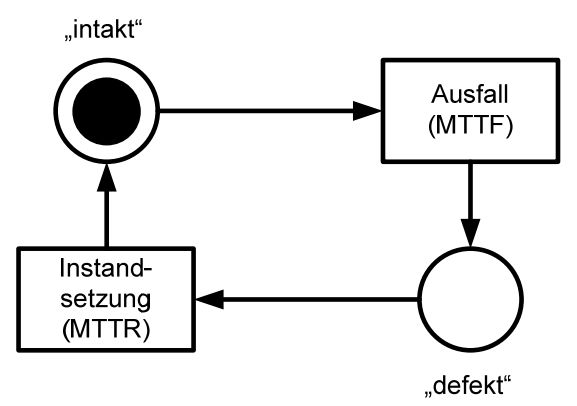

Abbildung 2: Basis-Petri-Netz zur Abbildung von Systemkomponenten

Für ein Petri-Netz, das $k$ Komponenten mit den zwei inhärenten Zuständen abbildet, erhält man für jeden Berechnungsschritt $i$ einen Vektor $s_{i} \in \mathrm{N}^{n}$ mit $n=2 k$ Stellen, der den Zustand des Petri-Netzes beschreibt. Die Stelle $(2 j-1)$ beschreibt dabei den Intaktzustand $z_{i j}^{+}$der j-ten Komponente in Schritt $i$, die Stelle $(2 j)$ ihren Defektzustand $z_{i j}^{-}$. Aufgrund der Tatsache, dass die beiden inhärenten Zustände komplementär sind, d.h. eine Komponente entweder "intakt" oder "defekt“ ist, ist es für die weiteren Untersuchungen ausreichend, lediglich die Intaktzustände $z_{i j}^{+} \mathrm{zu}$ betrachten. Folglich sind lediglich alle Stellen $(2 j-1)$ des Ergebnisvektors $s_{i}$ zu betrachten, der hierdurch auf den Vektor $s_{i}^{+} \in \mathrm{N}^{k}$ reduziert werden kann.

Das Ergebnis dieses Schritts ist eine formale Darstellung des Zustandsverlaufs der einzelnen Komponenten in Form von zeitabhängigen Vektoren. Die Summe über das Produkt aus der Intervalllänge $\Delta t_{i}$ und dem Zustandsindikator $z_{i}^{+}$ergibt die Anzahl der Intervalle, in der die Komponente „intakt“ war. In Verbindung mit der Summe über alle Intervalllängen lässt sich damit die Anzahl der Intervalle bestimmen, in denen die Komponente „defekt“ war. Hieraus lässt sich die Verfügbarkeit der jeweiligen Einzelkomponente berechnen.

\section{$3 \quad$ Ermittlung von Systemkennwerten}

Bevor die eigentliche Ermittlung der Systemkennwerte stattfinden kann, sind die strukturellen und logischen Abhängigkeiten der Systemkomponenten näher zu betrachten. Hierzu werden innerhalb des definierten Strukturgraphen im ersten Schritt alle Pfade durch das System gesucht. Das Ergebnis lässt sich in einer Pfadmatrix $P \in \mathrm{N}^{k \times k}$ darstellen, die Aufschluss über alle möglichen Pfade innerhalb des Systems gibt. Jeder Pfad wird dabei durch einen Zeilenvektor $p_{a} \in \mathrm{N}^{k}$ beschrieben, der Aufschluss darüber gibt, ob Komponente $j$ an Pfad $a$ beteiligt ist ( $\left.p_{a j}=1\right)$ oder nicht $\left(p_{a j}=0\right)$. Die Spaltenvektoren geben Aufschluss über die Anzahl der Pfade, an denen eine Komponente beteiligt ist. Je größer die Spaltensumme ist, umso kritischer ist die Komponente aus Sicht der Verfügbarkeit. Entscheidend für die Ermittlung der Verfügbarkeit sind jedoch die in der Steuerung des Systems realisierten Pfade. Diese lassen sich ebenfalls durch Pfadvektoren $c_{a} \in \mathrm{N}^{k}$ bzw. eine Pfadmatrix $C \in \mathrm{N}^{k \times k}$ beschreiben. Mit Hilfe der Pfadvektoren $c_{a}$ lässt sich im 
nächsten Schritt der Zustand $Z_{a}(i)$ des jeweiligen Pfades $a$ in Abhängigkeit des Berechnungsschritts $i$ gemäß Gleichung 1 bestimmen:

$$
Z_{a}(i)=\left\lfloor\frac{\sum_{j=1}^{k} c_{a j} z_{i j}^{+}}{\sum_{j=1}^{k} c_{a j}}\right\rfloor
$$

Ein Pfad ist demnach im Intaktzustand, falls alle beteiligten Komponenten sich im Intaktzustand befinden. Aus der Menge aller Zustandsindikatoren $Z_{a}(i)$ lässt sich der Zustand des Gesamtsystems $X(i)$ nach Gleichung 2 ermitteln:

$$
X(i)=\sum Z_{a}(i) \quad \text { mit } \quad 0 \leq X(i) \leq k
$$

Dieser Zustandsindikator $X(i)$ des Gesamtsystems gibt somit Aufschluss über die Anzahl der in Berechnungsschritt $i$ intakten Pfade. Auf dieser Grundlage lässt sich die Verfügbarkeit des Systems bestimmen. Hierzu ist vorab zu definieren, wie viele der Pfade im intakten Zustand sein müssen, damit sich das System in einem intakten Zustand befindet. Diese Mindestanzahl wird im Folgenden als Verfügbarkeitsbasis $b>0$ bezeichnet. Das System ist folglich als „intakt“ anzusehen, sofern gilt:

$$
X(i) \geq b
$$

Aus den Zustandsindikatoren $X(i)$ lässt sich schließlich die Anzahl der Intervalle bestimmen, in dem eine bestimmte Anzahl an Pfaden intakt war. Die Anzahl der Zeitintervalle $T_{u}$ in einem Berechnungsintervall $[x, y]: \forall x, y \in\{1, \ldots, \max (i)\}$, in denen mindestens $u$ von $v$ Pfaden, die in der Steuerung realisiert sind und sich im Intaktzustand befinden, kann allgemein durch die folgende Gleichung rekursiv ermittelt werden:

$$
T_{u}=\left(\sum_{i=x+1}^{y} \Delta t_{i}\left(\frac{[X(i)+1-u)]+[(X(i)+1-u) \operatorname{sgn}(X(i)+1-u)]}{2}\right)\right)-T_{u+1}
$$

Dabei muss die Bedingung $0 \leq u \leq v$ erfüllt sein und es müssen für alle Schritte identische Berechnungsintervallgrenzen gelten. Das Verfahren beginnt mit $u=v$. In den weiteren Iterationsschritten wird $u$ jeweils um 1 vermindert. Der Fall $u=0$ repräsentiert alle Zeitintervalle, in der mindestens 0 Komponenten "intakt" sind und somit die Summe aller betrachteten Zeitintervalle. Abbildung 3 veranschaulicht die Berechnung am Beispiel eines Systems mit 2 Pfaden in Abhängigkeit der Zeit.

Die Summe der schraffierten Flächeninhalte des jeweiligen Iterationsschritts veranschaulicht den dort ermittelten Wert von $T_{u}$. Die Anzahl der Intervalle, in denen genau $u$ Pfade „intakt“ sind, kann durch entsprechende Differenzbildung aus den ermittelten Werten für $T_{u}$ bestimmt werden. 

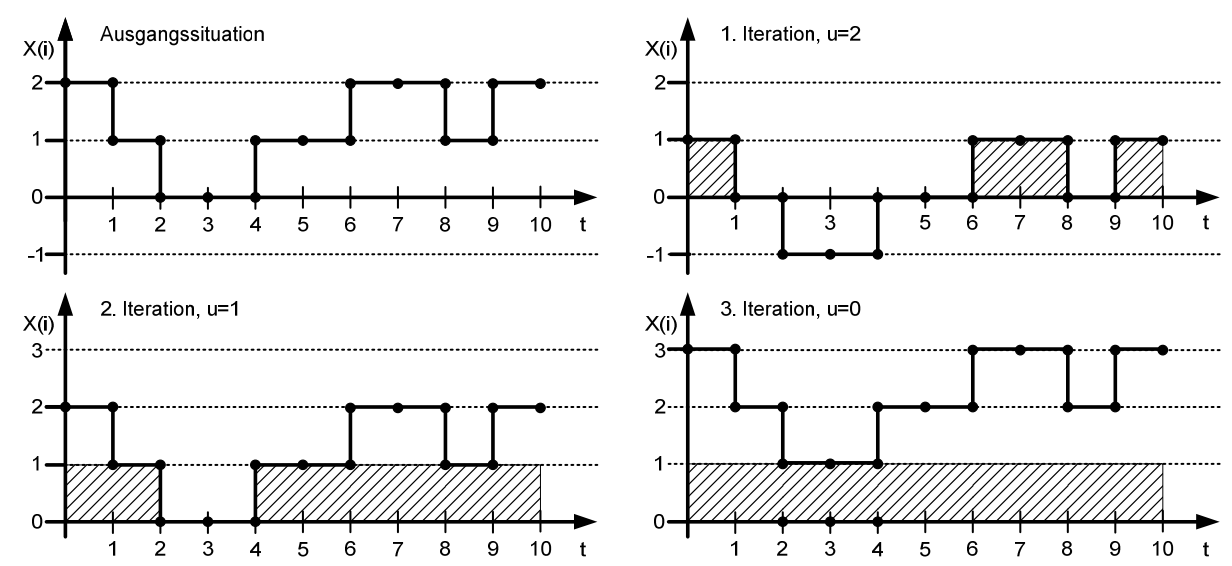

Abbildung 3: Graphische Veranschaulichung der Berechnung von $T_{u}$

Die Verfügbarkeit innerhalb des Berechnungsintervalls $[x, y]$ lässt sich in Abhängigkeit der Verfügbarkeitsbasis $b>0$ gemäß Gleichung 5 bestimmen:

$$
V=\frac{T_{b}}{T_{0}}
$$

Legt man für das Beispiel aus Abbildung 3 eine Verfügbarkeitsbasis von $b=1$ zugrunde, ergibt sich eine Verfügbarkeit von $80 \%$.

Zur Berechnung der Kenngrößen MTTF und MTTR ist die Anzahl der Ausfallereignisse $e^{-}$und die Anzahl der Instandsetzungen $e^{+} \mathrm{zu}$ ermitteln. Zur Identifikation der einzelnen Ereignisse sind Änderungen von $X$ (i) relevant, bei denen das System in den Defektzustand wechselt. Um diese ermitteln zu können, wird $X(i)$ in Verbindung mit der Verfügbarkeitsbasis $b$ gemäß Gleichung 6 zu $Y(i) \in[0,1]$ normiert $(X(i) \geq 0, b>0)$ :

$$
Y(i)=\operatorname{sgn}\left(\left\lfloor\frac{X(i)}{b}\right\rfloor\right)
$$

Der Zustandsindikator $Y(i)$ beschreibt folglich, ob das System in Berechnungsschritt $i$ intakt ist oder nicht. Um die einzelnen Ereignisse identifizieren zu können, wird nun die Änderung von $Y(i)$ zwischen zwei Berechnungsschritten gemäß Gleichung 7 ermittelt:

$$
\Delta Y(i)=Y(i)-Y(i-1): \forall i \in\{2, \ldots, \max (i)\}
$$

Alle negativen Werte von $\Delta Y(i)$ markieren ein Ausfallereignis, alle positiven eine Instandsetzung. Daraus lässt sich die Anzahl der jeweiligen Ereignisse $\mathrm{e}^{-}$und $\mathrm{e}^{+}$in den Intervallgrenzen $[x, y]: \forall x, y \in\{1, \ldots, \max (i)\}$ mit Hilfe der folgenden Gleichungen 8 und 9 bestimmen:

$$
\mathrm{e}^{-}=\sum_{i=x+1}^{y}\left|\frac{\Delta Y(i)-\Delta Y(i) \operatorname{sgn}(\Delta Y(i))}{2}\right|
$$




$$
\mathrm{e}^{+}=\sum_{i=x+1}^{y} \frac{\Delta Y(i)+\Delta Y(i) \operatorname{sgn}(\Delta Y(i))}{2}
$$

Aus diesen Größen lassen sich schließlich die MTTF gemäß Gleichung 10 bzw. die MTTR gemäß Gleichung 11 ermitteln:

$$
\operatorname{MTTF}=\frac{T_{b}}{\mathrm{e}^{-}} \quad \text { bzw. } \quad \text { MTTR }=\frac{T_{0}-T_{b}}{\mathrm{e}^{+}}
$$

Beide Kenngrößen lassen sich analog für einzelne Komponenten ermitteln. Hierzu können die Gleichungen 7 bis 11 mit den Parametern $z_{i}^{+}$und $b=1$ verwendet werden.

\section{$4 \quad$ Anwendung auf ein Systembeispiel}

Die in den vorherigen Abschnitten vorgestellten Berechnungsvorschriften werden im Folgenden beispielhaft zur Anwendung gebracht. Konkret erfolgt dies mit Hilfe einer zweistufigen Vorgehensweise, deren prinzipieller Ablauf in Abbildung 4 dargestellt ist.

Die Trennung zwischen der Abbildung des Komponentenverhaltens und der anschließende Aggregation zum Systemverhalten bietet zudem den Vorteil, dass Methode problemlos während der Betriebsphase angewendet werden kann. In diesem Fall ist es lediglich notwendig, Betriebs- und Instandsetzungszeiten der einzelnen Komponenten, beispielsweise mit Hilfe einer Betriebsdatenerfassung, zu dokumentieren.

\section{Stufe 1: Nachbilden des Verhaltens der}

Systemkomponenten im Zeitverlauf

Stufe 2: Aggregation des Komponentenverhaltens zum

Systemverhalten und Berechnung von Kennwerten

Ergebnis: Kennwerte der Komponenten und des

Systems im Zeitverlauf

Abbildung 4: Vorgehensmodell zur realitätsnahen Abbildung der Verfügbarkeit

Im konkreten Fall wird in der ersten Stufe das Verhalten der einzelnen Systemkomponenten im Zeitverlauf mit Hilfe von Petri-Netzen nachgebildet (vgl. Abschnitt 2). Mit Hilfe der Formeln des Abschnitts 3 wird das Komponentenverhalten zum Systemverhalten aggregiert, auf dessen Basis die entsprechenden Kennwerte ermittelt werden können. Nachdem die grundlegende Funktionsfähigkeit des Berechnungsverfahrens in [tHo09] nachgewiesen wurde, wird an dieser Stelle ein Modell mit höherer Komplexität gebildet und die Ergebnisse auf ihre Stabilität hin untersucht. Insbesondere aufgrund der ringförmigen Strukturen ist das gewählte Beispielsystem mit den etablierten Verfahren (vgl. Abschnitt 1) nicht modellierbar, da hierfür keine Strukturformeln bzw. Strukturelemente existieren. Der 
Materialflussgraph des Beispielsystems ist in Abbildung 5 dargestellt. Alle betrachteten Einzelkomponenten werden vereinfachend mit einer Verfügbarkeit von 0,998 angenommen, die sich aus einer $M T T F=500 \mathrm{ZE} \mathrm{bzw}$. MTTR $=1 \mathrm{ZE}$ errechnet.

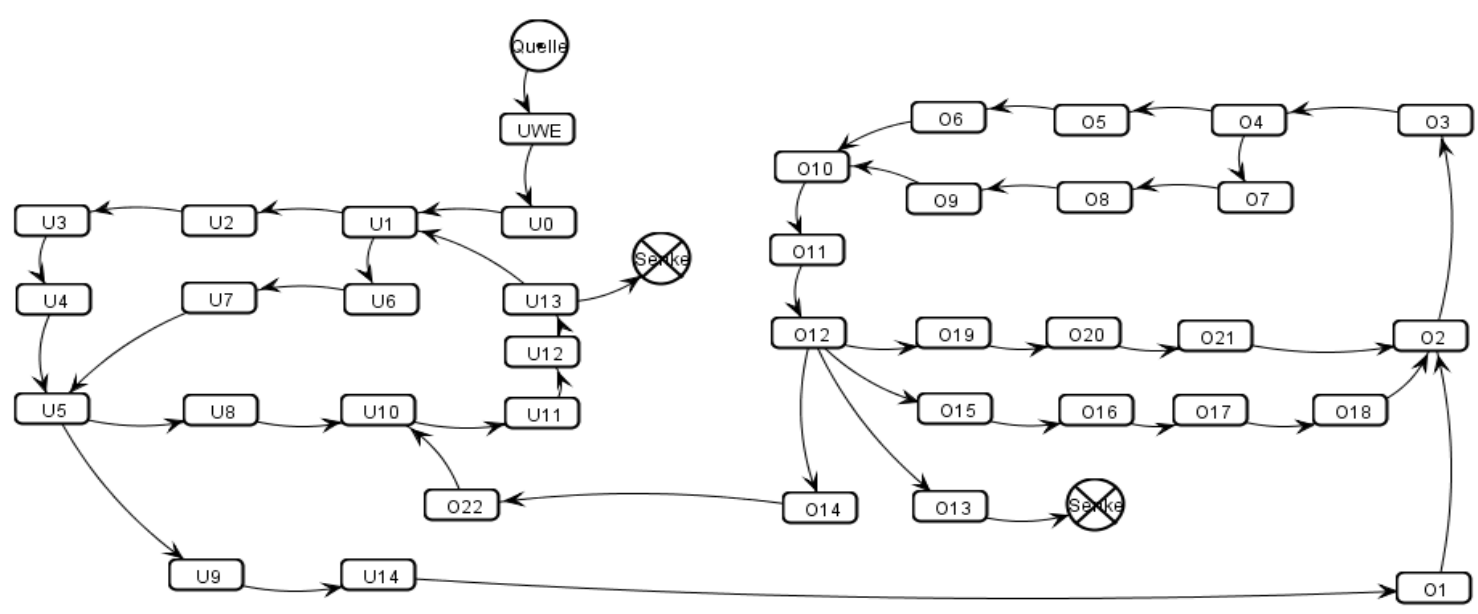

Abbildung 5: Materialflussgraph für ein existierendes Materialflusssystem

Um die Gesamtverfügbarkeit zu berechnen, werden nun zunächst alle möglichen Quelle-Senke-Beziehungen aus dem gegebenen Materialflussgraph ermittelt. Es ergeben sich vier bzw. sechs mögliche Pfade von der Quelle zu den zwei vorhandenen Senken und damit eine maximal mögliche Verfügbarkeitsbasis von 10. Die anschließende Berechnung der Verfügbarkeiten für die Basiswerte von eins bis zehn ergeben die in Tabelle 1 dargestellten Werte.

\begin{tabular}{|c|c|c|c|}
\hline Verfügbarkeitsbasis $b$ & Verfügbarkeit & MTTF [ZE] & MTTR [ZE] \\
\hline 1 & 0,992 & 191,96 & 1,58 \\
\hline 2 & 0,992 & 186,80 & 1,57 \\
\hline 3 & 0,974 & 60,80 & 1,61 \\
\hline 4 & 0,974 & 60,35 & 1,61 \\
\hline 5 & 0,966 & 46,58 & 1,62 \\
\hline 6 & 0,957 & 36,41 & 1,64 \\
\hline 7 & 0,942 & 26,97 & 1,66 \\
\hline 8 & 0,942 & 26,97 & 1,66 \\
\hline 9 & 0,940 & 26,20 & 1,67 \\
\hline 10 & 0,940 & 26,20 & 1,67 \\
\hline
\end{tabular}

Tabelle 1: Verfügbarkeiten des Beispielsystems

Es ist zu erkennen, dass die Gesamtverfügbarkeit mit zunehmender Verfügbarkeitsbasis abnimmt. Je höher dieser Wert gewählt wird, umso mehr Pfade müssen gemäß Definition gleichzeitig intakt sein, damit die gesamte Anordnung sich im intakten Zustand befindet. Aufgrund der Tatsache, dass bei der Ausführung der Petri-Netze Zufallszahlen zur Abbildung der stochastischen Effekte generiert werden, sind die ermittelten Ergebnisse in Bezug auf ihre Stabilität zu bewerten, $d$. h. es ist zu prüfen, inwieweit eine Wechselwirkung zwischen dem zufälligen Ausfallverhalten 
einzelner Komponenten und dem Gesamtsystem besteht. Hierzu wird der Zufallszahlengenerator, der das Ausfallverhalten der einzelnen Systemkomponenten steuert, mit unterschiedlichen Anfangswerten $r_{i}$ (sog. Seeds) gestartet. Dadurch ergeben sich deutliche Unterschiede in den Ausfallzeitpunkten und -dauern (vgl. Abbildung 6). Trotzdem nähert sich die Komponente mit zunehmender Laufzeit einem nahezu identischen Verfügbarkeitswert an.

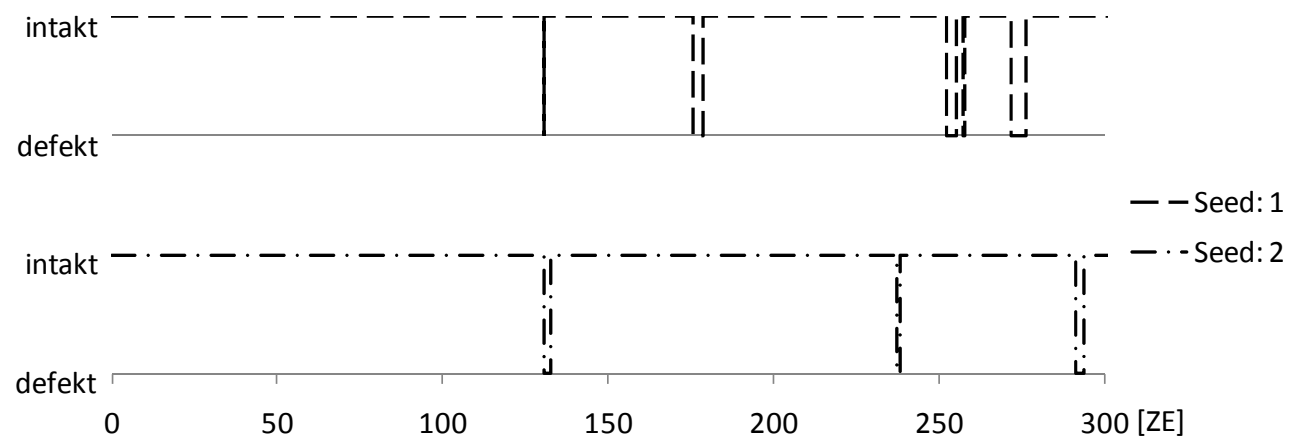

Abbildung 6: Beispielhaftes Ausfallverhalten einer Komponente

Repräsentativ werden für das Beispielsystem zehn Berechnungsläufe betrachtet, bei denen die Seeds $r_{i} \in\{1 \ldots 10\}$ verwendet werden. Die maximale Standardabweichung bei der Systemverfügbarkeit beträgt 0,024 \%, Der aus den zehn ermittelten Werten erkennbare Trend wird bei Erhöhung die Anzahl der betrachteten $r_{i}$ weiter verstärkt. Es ergeben sich folglich trotz unterschiedlichen Ausfallverhaltens der betrachteten Einzelkomponenten nur geringe Abweichungen der Systemverfügbarkeit. Dies gilt ebenfalls für die ermittelten Ausfall- und Instandsetzungsdauern.

Ein allgemeiner Nachweis der Ergebnisgüte kann mit Hilfe des Prinzips der „Ergodizität" erbracht werden, die im Bereich der Zeitreihenanalyse geprägt wurde. Sie basiert auf Beobachtungen zu verschiedenen Zeitpunkten, wobei die Beobachtungen als Realisation verschiedener abhängiger Zufallsvariablen aufgefasst werden (siehe [Sch97]). Hierbei werden die Mittelwerte für jede aufgenommene Datenreihe zu einem gegebenen Zeitpunkt als Scharmittelwerte und die Werte, die aus der Berechnung des Mittelwerts bezogen auf eine Zeitreihe resultieren, als Zeitmittelwert bezeichnet (vgl. Abbildung 7). Wenn diese beiden Werte gleich sind, liegt ein ergodischer Prozess vor und die Berechnung eines einzigen Zeitmittels ist ausreichend, um die Mittelwerte aller Zeitmittel zu bestimmen ([Kle06, S. 415] bzw. [Sch97, S.230]). Eine zentrale Voraussetzung für die Ergodizität ist die Stationarität des betrachteten Zufallsprozesses ([Hän07, S. 69] und [Sch97, S. 230]). Die Definition stationärer Prozesse besagt, dass berechenbare statistische Eigenschaften „invariant gegenüber Verschiebungen der Zeit sind“ [Hän07, S. 66], also beispielsweise der Mittelwert einer Zeitreihe unabhängig vom Startzeitpunkt bestimmt werden kann.

Im vorliegenden Fall ist diese Bedingung für alle Zeitpunkte $t$ gegeben, in denen sich die Berechnungen der hinterlegten Petri-Netze bereits eingependelt haben. Dort 
können Mittelwerte für unterschiedliche Startparameter $r_{i}$ gebildet werden, die sich nur noch unwesentlich unterscheiden.

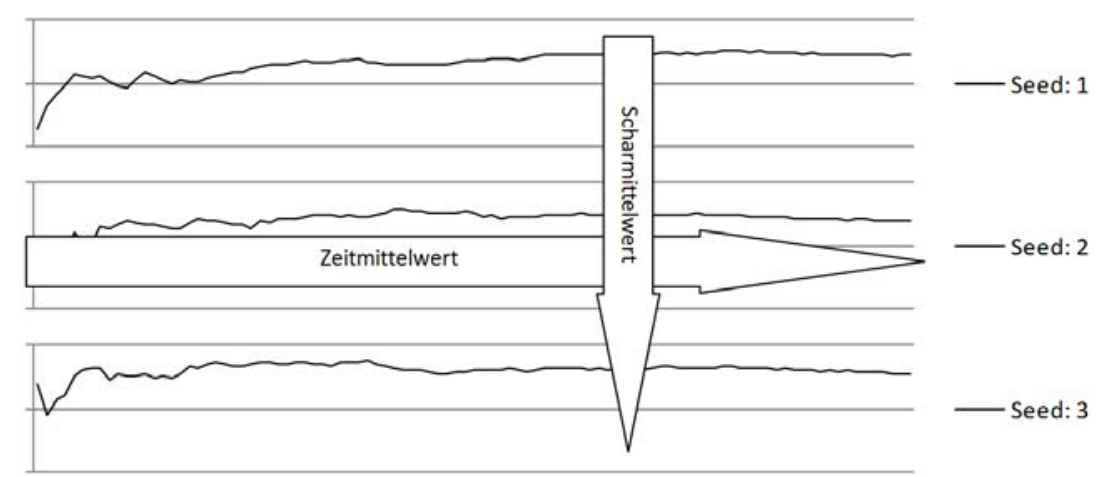

Abbildung 7: Darstellung Zeit- und Scharmittel (nach [Hän07, S. 59])

Die in [Hän06, S. 69ff.] vorgestellte Form des Beweises der Ergodizität ist aufgrund fehlender Abbildungs- und Berechnungsvorschriften der betrachteten Daten nicht vollständig übertragbar. Für den Fall des vorliegenden Berechnungsverfahrens kann also die Ergodizität nur „angenommen“ werden. Dies ist zulässig, da „es auch bei einem Zufallsprozess, der nicht ergodisch ist, einzelne Musterfunktionen gibt, die zumindest bezüglich der interessierenden Eigenschaft repräsentativ für den Prozess sind“ [Hän06, S. 71]. In weiteren Untersuchungen wird jedoch angestrebt, diesen Nachweis zu erbringen.

\section{$5 \quad$ Zusammenfassung und Ausblick}

Im Rahmen des vorliegenden Beitrags wurde eine Methode zur realitätsnahen Abbildung der technischen Verfügbarkeit intralogistischer Systeme vorgestellt. Die Methode gliedert sich in zwei Stufen: die realitätsnahe Abbildung des Verhaltens der einzelnen Komponenten und die anschließende Aggregation zum Verhalten des Systems, auf dessen Basis die Verfügbarkeit und verwandte Kenngrößen ermittelt werden können. Mit Hilfe eines Systembeispiels wurde die Anwendbarkeit auf komplexe Systemstrukturen sowie eine für praktische Anwendungen ausreichende Ergebnisstabilität gezeigt. Der Kennwert der Verfügbarkeit alleine ist jedoch keine ausreichende Bewertungsgrundlage für die tatsächlich nutzbare Leistung intralogistischer Systeme. Aus diesem Grund soll ein integratives Berechnungsmodell entwickelt werden, dass die Kennwerte Verfügbarkeit und Leistung miteinander kombiniert, ohne hierfür aufwändige Simulationsstudien durchführen zu müssen. Um dieses Ziel zu erreichen, sind insbesondere weiterführende Arbeiten zur Leistungsermittlung von Materialflusssystemen erforderlich. Die Anwendung der in diesem Bereich existierenden Verfahren ist vielfach auf einzelne Teilsysteme oder Systemelementen beschränkt. Die Wechselwirkungen zwischen einzelnen Systemelementen wurde bis dato trotz ihrer starken Verkettung nicht oder nur unzureichend berücksichtigt. Diese Problematik soll durch die Entwicklung geeigneter Methoden aufgelöst werden, sodass erstmals 
die Leistungsermittlung eines vollständigen Systems ermöglicht wird. In Verbindung mit der vorgestellten Methode der Verfügbarkeitsanalyse soll schließlich ein integratives Modell entstehen, mit dessen Hilfe die Leistungsverfügbarkeit im zeitlichen Verlauf ermittelt werden kann.

\section{Literatur}

[Arn90] Arnold, D.: Die Verfügbarkeit - Selbstzweck oder Kostenfaktor?, in VDI Berichte 833, VDI Verlag, Düsseldorf 1990

[Bov00] Bovert, E.-M. vom: Modellerstellung zur Verfügbarkeitsprognose komplexer Förder- und Lagersysteme, Dissertation, Universität Dortmund, 2000

[Fau08] Faulin, J. et al.: Predicting availability functions in time-dependent complex systems with SAEDES simulation algorithms. In: Reliability Engineering \& System Safety, Volume 93, Issue 11, Pages 1761-1771, November 2008

[Hän01] Hänsler, E.: Statistische Signale, Berlin Heidelberg: Springer Verlag, 2001

[Kle06] Klenke, A.: Wahrscheinlichkeitstheorie, Berlin Heidelberg New York: Springer Verlag, 2006

[Neb09] Nebel, S.; Dieter, A.; Bertsche, B.: Modellbasierte Optimierung der Instandhaltungsstrategie von Werkzeugmaschinen auf Basis farbiger Petrinetze mit Berücksichtigung ungenauer Ausfalldaten. In: VDI Berichte 2065, VDI Verlag, Düsseldorf 2009

[Poz06] Pozsgai, P.: Realitätsnahe Modellierung und Analyse der operativen Zuverlässigkeitskennwerte technischer Systeme, Dissertation, Universität Stuttgart, 2006

[Pri08] Priese, L.; Wimmel, H.: Petri-Netze, 2. Aufl.; Springer Verlag, Berlin 2008

[Sch97] Schlittgen, R.; Streitberg, B. H. J.: Zeitreihenanalyse, München Wien: R. Oldenbourg Verlag, 1997

[tHo07] ten Hompel, M.; Follert, G.; Jung, E.-N.: Modellierung des Verfügbarkeitsverhaltens von Intralogistiksystemen. In: Crostack, H.-A. (Hrsg.), ten Hompel, M. (Hrsg.):: Forderungsgerechte Auslegung von intralogistischen Systemen - Logistics on Demand Nr. 2, S. 143-162, Dortmund 2007

[tHo09] ten Hompel, M.; Jung, E.-N.; Feldhorst, S.: Modellierungs-ansatz für die realitätsnahe Abbildung der technischen Verfügbarkeit intralogistischer Systeme. In: Künne, B. (Hrsg.), Tillmann, W. (Hrsg.), Crostack, H.A.(Hrsg.): Forderungs-gerechte Auslegung von intralogistischen Systemen - Logistics on Demand Nr. 3, S. 159 - 179, Dortmund, 2009

[VDI04] VDI-Richtlinie 3581: Zuverlässigkeit und Verfügbarkeit von Transportund Lageranlagen, Beuth Verlag, Berlin, Dezember 2004 